\title{
An Analysis of Motivation of Students at Sports Science Faculty as Football Sports Fans
}

\author{
Emre Belli $^{1, *}$, Ali Gürbüz², Yunus Sinan Biricik ${ }^{1}$ \\ ${ }^{1}$ Department of Sport Management, Faculty of Sport Science Ataturk University, Turkey \\ ${ }^{2}$ Department of Physical Education and Sport, Rectorate, Mimar Sinan University, Turkey
}

Copyright $\mathrm{O} 2016$ by authors, all rights reserved. Authors agree that this article remains permanently open access under the terms of the Creative Commons Attribution License 4.0 International License

\begin{abstract}
The aim of this study is to analyze motivation of students at sports science faculty as football sports fans. To collect data about motivation of students for the current research, "Sport Fans Questionnaire" was used which was developed by Karaoğlu and Tiryaki [1] and it was administered to a total of 273 students consisting of 179 male and 94 female students. For data analysis, SPSS statistical packet program was used for frequency analysis, descriptive statistic, independent t-tests and one-way ANOVA. Tukey test was run to find out the source of the difference among different groups of participants. The analysis of fans motivations showed that there was not a statistically significant difference among participants from different departments in terms of seeking excitement-fun $(p=, 760)$, pride $(p=, 369)$, aggression $(p=, 093)$, closeness sympathy $(\mathrm{p}=, 060)$, understanding-knowing $(\mathrm{p}=, 327)$, and escape $(p=, 597)$. However, it was revealed that there was statistically significant difference among participants in aesthetic terms especially on behalf of students of physical education and sports. Based on these results, it can be seen that students at physical training and sports department were more motivated than the students of sports management department as a fan while watching match with regard to aesthetic dimension.
\end{abstract}

Keywords Fans, Motivation, Physical Education, Sport

\section{Introduction}

The concept of fans refers to taking and supporting one side. The word "fan", derived from the original word "fanatic", was used for the first time in 1682 to describe overly enthusiastic people [2]. In terms of football, this word was used to describe the groups who were attracted to the values represented by the players and supportive of the financial and moral activities of the football clubs. For example, a person who feels close to an individual, team, style of music, artist, and etc. [3]
When we have a look at the concept of fan base, nowadays, it has become synonymous with football fans to such an extent that we first relate this to football before any other type of fan base. Therefore, people believe that they become more popular once they become a fan especially when they are a fan of a football team. This mindset has overshadowed the other fan bases of Music, Art and other interests. When we look at the term "fan" and when it's related to football, we mean a person that is infatuated and interested in football and supports the football club. [4]

Currently, football fans have become an integral part of the football culture. Today it would not be incorrect to say that football has become a subculture with its own rhetoric and slang language and the fan-base are regarded as the driving force of this culture. In addition, fans of the popular football culture are enveloped into society and represent the establishment of a micro-economic community. [5]

Football fandom is connected to the team ethics and values which are reflected by the players, and they support the football club's activities in both a financial and morale sense by purchasing the clubs services for a specific fee in both domestic and international away games despite any hardships incurred. [6]

It can be simply said that being a fan is supporting, binding and having an allegiance to a specific football club. Football clubs constantly evaluate their position based on an increase or decrease in support of their fan base because the fan base is a group of people devoted to the team for a long period of time. The assessment of the club's value is also reflected by the number of their fan base. [3]

Human beings have an instinctive behavior to survive which is hidden deep within their genetic codes, this has evolved since the beginning of mankind like hunting, gathering and avoiding danger. These instinctive behaviors overshadowed by today's modern life and are urging to come to the surface. In this sense, the sports industry is a phenomenon that settled in human life as a result of that urge. For example, hunting is replaced by athleticism, self-defense is replaced by wrestling and gathering or being a part of the group and chasing a prey is replaced by football [7]. Sports 
Psychologist Ceren Toksöz explains the reason why football has had such an important role in our lives in her article entitled "Why do we love football?".

Football has become engrained in our genes and unfortunately the majority of the international fan base allows its primitive instincts to manifest themselves as violence and aggression while following the sport as if football and aggression were systemic which of course is not the case.

As claimed by evolutionary psychologists, people still can be very strongly in control of some of their primitive instincts, but what we should never forget is that we are now civilized beings living in the modern world. If this hadn't occurred, we wouldn't be able to walk safely in the street or we couldn't have this way of life. It has been noted that football related violence and aggression takes place in those countries which are of a lower socio-cultural level. As it is well known the augmentation of civilization and as a result the intellectual, economical, cultural and social upbringing will be developed and that development will help suppress and control the primitive instincts. At such locations, self-control is at the highest level and outside influences are at the lowest level. For example, if a stadium is filled with civilized and disciplined human beings, the less the need for police, security guards, fences, etc. But again we should also state that, because football is a sport watched as a group this creates a more favorable environment for violence and aggression. The reason is again the Herd Mentality and the "strength in numbers" syndrome. This group environment predisposes people to be more aggressive. For this reason, people that would not normally be predisposed to violence or aggression when in a group show unawaited behavior in direct conflict to their everyday character. [8]

You can find examples where football fans leave their daily lives behind and stand side by side with people of differing social stature and political views in the Stadium which would not happen anywhere else. It can also be noticed that the fans suppress their anger and frustration when they are in large groups in the stadium watching the Match. The feeling of being in a large group, the competitive feeling of the game and the possibility of surprises create a unique sense of belonging and lifelong Loyalty [8].

The reason why football has become a passion for people on many levels is that it nurtures our basic instincts. On the other hand, all the issues mentioned in this article which are resultant from the need to bond with the players, the teams and football, has created this large sub-culture [8].

To be able to understand how people who have studied sports have fanatical feelings for the club they support and understand what factors motivate them to go to their teams' matches is significant in terms of having an idea of the impact of education on fanaticism.

\section{Material and Methods}

This study was designed to investigate motivations of students at sports science faculty as football sports fans.

The population of the research was comprised of the students at the Faculty of Sports Sciences at Atatürk University and the sample for the current study were consisted of a total of 273 students that included 179 male students and 94 female students.

For data collection, the questionnaire called "Sport Fans Questionnaire" that was developed by Karaoğlu and Tiryaki [1] was used to gather data. For data analysis, SPSS statistical packet program was used and the significance level was accepted as $(p<0,05)$.

In the current research, frequency analysis was performed for the analysis of demographic features of the students, descriptive statistics was used to find out the general averages, independent samples $t$-tests were run to compare the students' motivation as football sports fans in terms of gender and type of branch, one-way ANOVA was carried out to find out the difference between motivation as football sports fans in terms of age, grade, team of fans and the department and lastly, Tukey test was run to find out from which group the difference results.

\section{Findings}

Considering the distribution of participants in terms of gender, it is obvious that $65,6 \%$ of them are men and $34,4 \%$ are women. Besides, when we look at the branches, we see that $63 \%$ of them are interested in team sports and $37 \%$ of them prefer individual sports. As for the age, we can see that $26,7 \%$ of them are between the ages of 20 and below, $68,9 \%$ of them are between 21-25 ages and 4,4\% are 26 and above ages. Regarding the distribution of participants in terms of branches, it is obvious that $24,2 \%$ of them are in PE and Sports Academy, 24,9\% are in Sports Management, 26,7\% of them are in Coaching and $24,2 \%$ are the students of Recreation. As for the grade variable, it is revealed that $17,2 \%$ of the participants are in first grade, $36,3 \%$ are in second grade, $21,2 \%$ are in third grade and $25,3 \%$ of them are in fourth grade. Checking out the favorite teams of participants, the following percentages of participants were observed to belong to each football team: $39,9 \%$ as Galatasaray, 25,3\% as Fenerbahçe and 19,4\% as Beşiktaş. The other teams are supported by $15,4 \%$. 
Table 1. Data on the Demographic Features of the Participants

\begin{tabular}{|c|c|c|}
\hline Gender & $\mathrm{N}$ & \multicolumn{1}{|c|}{$\%$} \\
\hline Men & 179 & 34,4 \\
\hline Woman & 94 & $\%$ \\
\hline Branch & $\mathrm{N}$ & 33,0 \\
\hline Team Sports & 172 & 37,0 \\
\hline Individual Sports & 101 & 26,7 \\
\hline Age & $\mathrm{N}$ & 68,9 \\
\hline 20 years and below & 73 & 4,4 \\
\hline $21-25$ years & 188 & $\%$ \\
\hline 26 and above & 12 & 24,2 \\
\hline Department & $\mathrm{N}$ & 24,9 \\
\hline P.E and Sports teaching & 66 & 26,7 \\
\hline Sports management & 68 & 24,2 \\
\hline Coach training & 73 & $\%$ \\
\hline Recreation & 66 & 17,2 \\
\hline Class & $\mathrm{N}$ & 36,3 \\
\hline 1st grade & 47 & 21,2 \\
\hline 2nd grade & 99 & 25,3 \\
\hline 3rd grade & 58 & $\%$ \\
\hline 4th grade & 69 & 19,4 \\
\hline Team & $\mathrm{N}$ & 25,3 \\
\hline Beşiktaş & 53 & 39,9 \\
\hline Fenerbahçe & 69 & 15,4 \\
\hline Galatasaray & 109 & 100 \\
\hline Others & 42 & \\
\hline Total & 273 & \\
\hline
\end{tabular}

Table 2. The Analysis of Motivation of Sports Fans Related to the Gender of Participants

\begin{tabular}{|c|c|c|c|c|c|c|}
\hline Subscale & Gender & $\mathrm{N}$ & Mean & Std. Deviation & $\mathrm{T}$ & $\mathrm{P}$ (sig.) \\
\hline Seeking excitement/fun & $\begin{array}{l}\text { Male } \\
\text { Female }\end{array}$ & $\begin{array}{c}179 \\
94\end{array}$ & $\begin{array}{l}3,06 \\
2,84\end{array}$ & $\begin{array}{l}, 855 \\
, 926\end{array}$ & 1,972 &, 233 \\
\hline Pride & $\begin{array}{c}\text { Male } \\
\text { Female }\end{array}$ & $\begin{array}{c}179 \\
94 \\
\end{array}$ & $\begin{array}{l}3,12 \\
2,96\end{array}$ & $\begin{array}{l}, 967 \\
1,065 \\
\end{array}$ & 1,212 & ,109 \\
\hline Aggression & $\begin{array}{l}\text { Male } \\
\text { Female }\end{array}$ & $\begin{array}{c}179 \\
94\end{array}$ & $\begin{array}{l}2,64 \\
2,45\end{array}$ & $\begin{array}{l}1,004 \\
1,052\end{array}$ & 1,415 &, 331 \\
\hline Closeness sympathy & $\begin{array}{l}\text { Male } \\
\text { Female }\end{array}$ & $\begin{array}{c}179 \\
94\end{array}$ & $\begin{array}{l}2,94 \\
2,54\end{array}$ & $\begin{array}{l}, 876 \\
, 891\end{array}$ & 3,498 &, 811 \\
\hline Aesthetic & $\begin{array}{c}\text { Male } \\
\text { Female }\end{array}$ & $\begin{array}{c}179 \\
94 \\
\end{array}$ & $\begin{array}{l}3,18 \\
3,07\end{array}$ & $\begin{array}{l}, 920 \\
1,056\end{array}$ &, 817 &, 076 \\
\hline Understanding-knowing & $\begin{array}{c}\text { Male } \\
\text { Female }\end{array}$ & $\begin{array}{c}179 \\
94 \\
\end{array}$ & $\begin{array}{l}3,02 \\
2,92\end{array}$ & $\begin{array}{l}, 957 \\
1,039\end{array}$ & ,792 &, 380 \\
\hline Escape & $\begin{array}{l}\text { Male } \\
\text { Female }\end{array}$ & $\begin{array}{c}179 \\
94\end{array}$ & $\begin{array}{l}2,82 \\
2,42\end{array}$ & $\begin{array}{l}, 881 \\
, 881\end{array}$ & 3,610 &, 937 \\
\hline
\end{tabular}

Based on the findings of data analysis, each category of motivation variables was found to have the following significance levels: seeking excitement/fun $(\mathrm{p}=, 233)$, pride $(\mathrm{p}=, 109)$ aggression $(\mathrm{p}=, 331)$ Closeness sympathy $(\mathrm{p}=, 811)$, aesthetic $(\mathrm{p}=, 0,76)$, understanding and knowing $(\mathrm{p}=, 380)$ and escape $(\mathrm{p}=, 937)$, which means that there were no significant differences between females and males regarding motivation of sports fans.

Table 3. The Analysis of Motivation of Sports Fans Related to Team Genres

\begin{tabular}{|c|c|c|c|c|c|c|}
\hline Subscale & Teams & $\mathrm{N}$ & Mean & Std. Deviation & $\mathrm{T}$ & $\mathrm{P}$ (sig.) \\
\hline Seeking excitement/fun & $\begin{array}{c}\text { Team } \\
\text { Individual }\end{array}$ & $\begin{array}{l}172 \\
101\end{array}$ & $\begin{array}{l}3,06 \\
2,84\end{array}$ & $\begin{array}{l}, 845 \\
, 936\end{array}$ & 1,989 &, 173 \\
\hline Pride & $\begin{array}{c}\text { Team } \\
\text { Individual }\end{array}$ & $\begin{array}{l}172 \\
101 \\
\end{array}$ & $\begin{array}{l}3,11 \\
2,98 \\
\end{array}$ & $\begin{array}{l}968 \\
1,057\end{array}$ & 1,063 & ,214 \\
\hline Aggression & $\begin{array}{c}\text { Team } \\
\text { Individual }\end{array}$ & $\begin{array}{l}172 \\
101 \\
\end{array}$ & $\begin{array}{l}2,62 \\
2,50\end{array}$ & $\begin{array}{l}1,006 \\
1,051 \\
\end{array}$ & ,932 & ,386 \\
\hline Closeness sympathy & $\begin{array}{c}\text { Team } \\
\text { Individual }\end{array}$ & $\begin{array}{l}172 \\
101\end{array}$ & $\begin{array}{l}2,83 \\
2,76\end{array}$ & $\begin{array}{l}, 880 \\
, 935\end{array}$ & ,621 & ,802 \\
\hline Aesthetic & $\begin{array}{c}\text { Team } \\
\text { Individual }\end{array}$ & $\begin{array}{l}172 \\
101\end{array}$ & $\begin{array}{l}3,15 \\
3,12\end{array}$ & $\begin{array}{c}931 \\
1,031\end{array}$ & ,268 & ,209 \\
\hline Understanding-knowing & $\begin{array}{c}\text { Team } \\
\text { Individual }\end{array}$ & $\begin{array}{l}172 \\
101 \\
\end{array}$ & $\begin{array}{l}3,05 \\
2,87 \\
\end{array}$ & $\begin{array}{l}1,016 \\
, 925\end{array}$ & 1,439 &, 337 \\
\hline Escape & $\begin{array}{c}\text { Team } \\
\text { Individual }\end{array}$ & $\begin{array}{l}172 \\
101\end{array}$ & $\begin{array}{l}2,73 \\
2,61 \\
\end{array}$ & $\begin{array}{l}, 869 \\
, 950 \\
\end{array}$ & 1,086 &, 113 \\
\hline
\end{tabular}


As illustrated above, the comparison between teams and individuals in terms of each sub-scale of motivation, the following findings were obtained based on data analysis: Seeking excitement/fun $(p=, 173)$ pride $(p=, 214)$ aggression $(p=, 386)$ closeness sympathy $(\mathrm{p}=, 802)$, aesthetic $(\mathrm{p}=, 209)$, understanding and knowing $(\mathrm{p}=, 337)$ and escape $(\mathrm{p}=, 113)$. These findings demonstrated that there was no statistically significant difference among sports fans as team and individuals.

Table 4. Comparison of Differences Aimed at Identifying the Motives of Sports Fans According to the Ages of the Participants.

\begin{tabular}{|c|c|c|c|c|c|c|}
\hline Sub-scale & Age & $\mathrm{N}$ & Mean & S.D & $\mathrm{F}$ & $P($ sig. $)$ \\
\hline Seeking excitement/fun & $\begin{array}{l}20 \text { and under } \\
21-25 \\
26 \text { and above }\end{array}$ & $\begin{array}{c}73 \\
188 \\
12\end{array}$ & $\begin{array}{l}2,94 \\
3,01 \\
2,93\end{array}$ & $\begin{array}{l}, 856 \\
, 889 \\
.875\end{array}$ & ,872 & ,456 \\
\hline Pride & $\begin{array}{l}20 \text { and under } \\
21-25 \\
26 \text { and above }\end{array}$ & $\begin{array}{c}73 \\
188 \\
12 \\
\end{array}$ & $\begin{array}{l}3,30 \\
3,26 \\
3,23 \\
\end{array}$ & $\begin{array}{c}, 867 \\
1,045 \\
, 977 \\
\end{array}$ & ,863 & ,461 \\
\hline Aggressiveness & $\begin{array}{l}20 \text { and under } \\
21-25 \\
26 \text { and above }\end{array}$ & $\begin{array}{c}73 \\
188 \\
12\end{array}$ & $\begin{array}{l}2,62 \\
3.61 \\
3.56\end{array}$ & $\begin{array}{l}1,031 \\
1,016 \\
1,189\end{array}$ & ,082 & ,970 \\
\hline Closeness sympathy & $\begin{array}{c}20 \text { and under } \\
21-25 \\
26 \text { and above }\end{array}$ & $\begin{array}{c}73 \\
188 \\
12\end{array}$ & $\begin{array}{l}2,73 \\
2,85 \\
2,51\end{array}$ & $\begin{array}{l}, 899 \\
, 905 \\
, 775\end{array}$ & ,930 & ,437 \\
\hline Aesthetics & $\begin{array}{l}20 \text { and under } \\
21-25 \\
26 \text { and above }\end{array}$ & $\begin{array}{c}73 \\
188 \\
12 \\
\end{array}$ & $\begin{array}{l}3,13 \\
3,16 \\
3,00 \\
\end{array}$ & $\begin{array}{l}967 \\
, 968 \\
, 978 \\
\end{array}$ &, 713 &, 545 \\
\hline Understanding-Knowing & $\begin{array}{l}20 \text { and under } \\
21-25 \\
26 \text { and above }\end{array}$ & $\begin{array}{c}73 \\
188 \\
12 \\
\end{array}$ & $\begin{array}{l}2,92 \\
2,99 \\
3,27 \\
\end{array}$ & $\begin{array}{r}, 977 \\
, 984 \\
1,121 \\
\end{array}$ & ,407 &, 748 \\
\hline Escape & $\begin{array}{l}20 \text { and under } \\
21-25 \\
26 \text { and above }\end{array}$ & $\begin{array}{c}73 \\
188 \\
12\end{array}$ & $\begin{array}{l}2,58 \\
2,73 \\
2,75\end{array}$ & $\begin{array}{l}, 930 \\
, 884 \\
1,030\end{array}$ & 857 & ,464 \\
\hline
\end{tabular}

$*: \mathrm{p}<0,05$

Once the data was analyzed, significant difference was not spotted in the subscales of seeking excitement/fun ( $\mathrm{p}=, 456)$, pride $(\mathrm{p}=, 461)$, aggressiveness $(\mathrm{p}=, 970)$, closeness sympathy $(\mathrm{p}=, 437)$, aesthetics $(\mathrm{p}=, 545)$, understanding-knowing $(\mathrm{p}=, 748)$ and escape $(\mathrm{p}=, 464)$.

Table 5. Comparison of Differences Aimed at Identifying the Motives of Sports Fans According to the Departments of the Participants.

\begin{tabular}{|c|c|c|c|c|c|c|}
\hline Sub-scale & Department & $\mathbf{N}$ & Mean & SD & $\mathbf{F}$ & P(sig.) \\
\hline Seeking excitement/fun & $\begin{array}{l}\text { Teaching } \\
\text { Managing } \\
\text { Coaching } \\
\text { Recreation }\end{array}$ & $\begin{array}{l}66 \\
68 \\
73 \\
66 \\
\end{array}$ & $\begin{array}{l}3,02 \\
2,98 \\
3,04 \\
2,89 \\
\end{array}$ & $\begin{array}{l}, 917 \\
, 844 \\
, 880 \\
, 909 \\
\end{array}$ & ,391 & ,760 \\
\hline Pride & $\begin{array}{l}\text { Teaching } \\
\text { Managing } \\
\text { Coaching } \\
\text { Recreation }\end{array}$ & $\begin{array}{l}66 \\
68 \\
73 \\
66 \\
\end{array}$ & $\begin{array}{l}3,29 \\
3,24 \\
3,23 \\
3,26 \\
\end{array}$ & $\begin{array}{c}, 945 \\
, 987 \\
1,006 \\
1,067 \\
\end{array}$ & 1,055 &, 369 \\
\hline Aggressiveness & $\begin{array}{c}\text { Teaching } \\
\text { Managing } \\
\text { Coaching } \\
\text { Recreation }\end{array}$ & $\begin{array}{l}66 \\
68 \\
73 \\
66 \\
\end{array}$ & $\begin{array}{l}3,25 \\
3,27 \\
3,24 \\
3,30 \\
\end{array}$ & $\begin{array}{c}, 987 \\
, 934 \\
1,029 \\
1,106 \\
\end{array}$ & 2,160 & ,093 \\
\hline Closeness sympathy & $\begin{array}{c}\text { Teaching } \\
\text { Sports man. } \\
\text { Coaching } \\
\text { Recreation }\end{array}$ & $\begin{array}{l}66 \\
68 \\
73 \\
66 \\
\end{array}$ & $\begin{array}{l}2,96 \\
2,60 \\
2,93 \\
2,71 \\
\end{array}$ & $\begin{array}{l}, 904 \\
, 807 \\
, 891 \\
, 962 \\
\end{array}$ & 2,497 &, 060 \\
\hline Aesthetics & $\begin{array}{c}\text { Teaching } \\
\text { Managing } \\
\text { Coaching } \\
\text { Recreation. }\end{array}$ & $\begin{array}{l}66 \\
68 \\
73 \\
66 \\
\end{array}$ & $\begin{array}{l}3,38 \\
2,91 \\
3,21 \\
3,07 \\
\end{array}$ & $\begin{array}{l}, 989 \\
, 909 \\
, 936 \\
, 998 \\
\end{array}$ & 2,908 &, $035^{*}$ \\
\hline Understanding-Knowing & $\begin{array}{l}\text { Teaching } \\
\text { Managing } \\
\text { Coaching } \\
\text { Recreation }\end{array}$ & $\begin{array}{l}66 \\
68 \\
73 \\
66 \\
\end{array}$ & $\begin{array}{l}3,05 \\
2,98 \\
3,09 \\
2,80 \\
\end{array}$ & $\begin{array}{c}1,106 \\
, 928 \\
, 941 \\
, 959 \\
\end{array}$ & 1,157 &, 327 \\
\hline Escape & $\begin{array}{l}\text { Teaching } \\
\text { Managing } \\
\text { Coaching } \\
\text { Recreation }\end{array}$ & $\begin{array}{l}66 \\
68 \\
73 \\
66 \\
\end{array}$ & $\begin{array}{l}2,70 \\
2,59 \\
2,79 \\
2,64\end{array}$ & $\begin{array}{l}, 949 \\
, 758 \\
, 957 \\
, 928 \\
\end{array}$ & ,628 &, 597 \\
\hline
\end{tabular}

$*: \mathrm{p}<0,05$ 
Table 6. The Results of the Multiple Comparisons about the Ideas about Motives of Sports Fans According to the Departments of the Participants.

\begin{tabular}{|c|c|c|c|c|}
\hline \multirow[b]{2}{*}{ Sub Scale } & \multicolumn{4}{|c|}{ Post Hoc (Tukey Test) } \\
\hline & $\mathrm{Com}$ & & $\begin{array}{c}\text { Difference } \\
\text { between the } \\
\text { average }\end{array}$ & Significance \\
\hline Aesthetics & P.E and Sports teaching & $\begin{array}{l}\text { Sports Manage. } \\
\text { Coaching } \\
\text { Recreation }\end{array}$ & $\begin{array}{l}469 \\
, 173 \\
, 254\end{array}$ & $\begin{array}{l}, 025^{*} \\
, 709 \\
, 254\end{array}$ \\
\hline
\end{tabular}

$*: \mathrm{p}<0.05$

Table. 7. Comparison of Differences Aimed at Identifying the Motives of Sports Fans According to The Grades of The Participants.

\begin{tabular}{|c|c|c|c|c|c|c|}
\hline Subdimensions & Grade & $\mathbf{N}$ & Mean & S.D & $\mathbf{F}$ & P(sig.) \\
\hline Seeking excitement/fun & $\begin{array}{l}1^{\text {st }} \text { Grade } \\
2^{\text {nd }} \text { Grade } \\
3^{\text {rd }} \text { Grade } \\
4^{\text {th }} \text { Grade }\end{array}$ & $\begin{array}{l}47 \\
99 \\
58 \\
69\end{array}$ & $\begin{array}{l}2,70 \\
2,96 \\
3,08 \\
3,12\end{array}$ & $\begin{array}{l}, 958 \\
, 900 \\
, 795 \\
, 851\end{array}$ & 2,498 &, 060 \\
\hline Pride & $\begin{array}{l}1^{\text {st }} \text { Grade } \\
2^{\text {nd }} \text { Grade } \\
3^{\text {rd }} \text { Grade } \\
4^{\text {th }} \text { Grade }\end{array}$ & $\begin{array}{l}47 \\
99 \\
58 \\
69\end{array}$ & $\begin{array}{l}3,35 \\
3,28 \\
3,24 \\
3,17\end{array}$ & $\begin{array}{c}1,003 \\
1,035 \\
1,001 \\
970\end{array}$ &, 311 &, 817 \\
\hline Aggressiveness & $\begin{array}{l}1^{\text {st }} \text { Grade } \\
2^{\text {nd }} \text { Grade } \\
3^{\text {rd }} \text { Grade } \\
4^{\text {th }} \text { Grade }\end{array}$ & $\begin{array}{l}47 \\
99 \\
58 \\
69\end{array}$ & $\begin{array}{l}3,88 \\
3,60 \\
3,56 \\
3,28 \\
\end{array}$ & $\begin{array}{c}1,021 \\
, 940 \\
1,050 \\
1,064\end{array}$ & 3,222 &, $023^{*}$ \\
\hline Closeness Sympathy & $\begin{array}{l}1^{\text {st }} \text { Grade } \\
2^{\text {nd }} \text { Grade } \\
3^{\text {rd }} \text { Grade } \\
4^{\text {th }} \text { Grade }\end{array}$ & $\begin{array}{l}47 \\
99 \\
58 \\
69\end{array}$ & $\begin{array}{l}2,57 \\
2,76 \\
2,84 \\
2,99\end{array}$ & $\begin{array}{l}, 972 \\
, 858 \\
, 944 \\
, 845\end{array}$ & 2,155 &, 094 \\
\hline Aesthetics & $\begin{array}{l}1^{\text {st }} \text { Grade } \\
2^{\text {nd }} \text { Grade } \\
3^{\text {rd }} \text { Grade } \\
4^{\text {th }} \text { Grade }\end{array}$ & $\begin{array}{l}47 \\
99 \\
58 \\
69\end{array}$ & $\begin{array}{l}2,97 \\
3,10 \\
3,07 \\
3,37\end{array}$ & $\begin{array}{c}1,082 \\
, 927 \\
, 975 \\
, 915\end{array}$ & 1,984 &, 117 \\
\hline Understanding-Knowing & $\begin{array}{l}1^{\text {st }} \text { Grade } \\
2^{\text {nd }} \text { Grade } \\
3^{\text {rd }} \text { Grade } \\
4^{\text {th }} \text { Grade }\end{array}$ & $\begin{array}{l}47 \\
99 \\
58 \\
69\end{array}$ & $\begin{array}{l}2,70 \\
2,92 \\
3,06 \\
3,20\end{array}$ & $\begin{array}{l}1,035 \\
, 948 \\
, 955 \\
993\end{array}$ & 2,703 &, $046^{*}$ \\
\hline Escape & $\begin{array}{l}1^{\text {st }} \text { Grade } \\
2^{\text {nd }} \text { Grade } \\
3^{\text {rd }} \text { Grade } \\
4^{\text {th }} \text { Grade }\end{array}$ & $\begin{array}{l}47 \\
99 \\
58 \\
69\end{array}$ & $\begin{array}{l}2,40 \\
2,63 \\
2,83 \\
2,84\end{array}$ & $\begin{array}{l}, 844 \\
, 869 \\
, 984 \\
, 870\end{array}$ & 2,930 & ,034* \\
\hline
\end{tabular}

$*: \mathrm{p}<0,05$

While there was statistically significance difference in the sub-scale of aesthetics $(p=0,35)$, no significance difference was obtained in the sub-scales of seeking excitement/fun $(p=, 760)$, pride $(p=, 369)$, aggressiveness $(p=, 093)$, closeness sympathy $(\mathrm{p}=, 060)$, understanding-knowing $(\mathrm{p}=, 327)$ and escape $(\mathrm{p}=, 597)$. The results of the multiple comparisons can be seen in Table 6 .

According to the multiple comparison test results, there was a statistically significant difference between Physical Education, Sports Teaching and Sports Management $(p=, 025)$ in the sub-scale of aesthetics $(p<0,05)$. According to this statistics, it was found out that students studying at the
Department of Physical Education and Sports Teaching ( $\bar{X}$ $=3,38 \pm, 989$ ) were found to give prominence to aesthetics while watching games more than the students studying at the Department of Sports Management ( $\bar{X}=2,91 \pm, 909)$.

Based on the analysis of data, significant difference was found in Aggressiveness $(p=, 023)$, Understanding-Knowing $(p=, 046)$ and Escape $(p=, 034)$. However, there was no significant difference $(\mathrm{p}<0,05)$ in the sub-scales of Seeking excitement/ Entertainment $(p=, 060)$, Pride $(p=, 817)$, Closeness Sympathy $(p=, 094)$ and Aesthetics $(p=, 117)$. The results of the multiple comparisons are displayed in Table 8 . 
Table 8. The Results of the Multiple Comparison about the Ideas about Motives of Sports Fans According to The Grades of the Participants.

\begin{tabular}{|c|c|c|c|c|}
\hline \multirow[b]{2}{*}{$\begin{array}{c}\text { Sub } \\
\text { Scale }\end{array}$} & \multicolumn{4}{|c|}{ Post Hoc (Tukey Test) } \\
\hline & Con & & $\begin{array}{c}\text { Difference } \\
\text { between the } \\
\text { average }\end{array}$ & Significance \\
\hline Aggressiveness & $1^{\text {st }}$ Grade & $\begin{array}{l}2^{\text {nd }} \text { Grade } \\
3^{\text {rd }} \text { Grade } \\
4^{\text {th }} \text { Grade }\end{array}$ & $\begin{array}{l}, 282 \\
, 324 \\
, 602 \\
\end{array}$ & $\begin{array}{l}, 952 \\
, 898 \\
, 022^{*} \\
\end{array}$ \\
\hline Understanding-Knowing & $4^{\text {th }}$ Grade & $\begin{array}{l}1^{\text {st }} \text { Grade } \\
2^{\text {nd }} \text { Grade } \\
3^{\text {rd }} \text { Grade }\end{array}$ & $\begin{array}{l}, 499 \\
, 282 \\
, 141\end{array}$ & $\begin{array}{l}, 037^{*} \\
, 256 \\
, 847\end{array}$ \\
\hline Escape & $4^{\text {th }}$ Grade & $\begin{array}{l}1^{\text {st }} \text { Grade } \\
2^{\text {nd }} \text { Grade } \\
3^{\text {rd }} \text { Grade }\end{array}$ & $\begin{array}{l}, 436 \\
, 209 \\
, 004\end{array}$ & $\begin{array}{l}, 049^{*} \\
, 441 \\
1,000\end{array}$ \\
\hline
\end{tabular}

$*: \mathrm{p}<0,05$

Table 9. Determining the Sports Fans Motives According to Sub Dimension Points that Were Taken From the Scale.

\begin{tabular}{|c|c|c|c|c|c|c|}
\hline Sub Dimensions & N & Min. & Max. & Mean & S.D \\
\hline Seeking excitement/fun & 273 & 1 & 5 & 2,98 &, 884 \\
\hline Pride & 273 & 1 & 5 & 3,26 & 1,002 \\
\hline Aggression & 273 & 1 & 5 & 3,58 & 1,022 \\
\hline Closeness Sympathy & 273 & 1 & 5 & 2,80 &, 899 &, 968 \\
\hline Aesthetics & 273 & 1 & 5 & 3,14 &, 985 \\
\hline Understanding and knowing & 273 & 1 & 5 & 2,98 & &, 900 \\
\hline Escape & 273 & 1 & 5 & & 2,68 & \\
\hline
\end{tabular}

According to the multiple comparison results, there was a significant difference $(p<0,05)$ in the sub-dimension of Aggressiveness between the $1^{\text {st }}$ and $4^{\text {th }}$ Grade $(p=, 022)$ students. According to that finding, it was found out that students studying in $1^{\text {st }}$ Grade $(\bar{X}=3,88 \pm 1,021)$ show more aggressive attitude while watching games compared to students studying in the $4^{\text {th }}$ Grade $(\bar{X}=3,28 \pm 1,064)$

There was a significant difference in the sub-scale of Understanding-Knowing $(\mathrm{p}<0,05)$ between the $1^{\text {st }}$ and $4^{\text {th }}$ Grade students $(p=, 037)$. According to that finding, it was found out that students studying in $4^{\text {th }}$ Grade $(\bar{X}=3,20 \pm, 993)$ value the sub-scale of Understanding-Knowing while watching games more than the students studying in the $1^{\text {st }}$ Grade ( $\overline{\mathrm{X}}=2,70 \pm 1,035)$.

There was a significant difference in the sub-scale of Escape $(\mathrm{p}<0,05)$ between the $1^{\text {st }}$ and $4^{\text {th }}$ Grade students $(p=, 049)$. According to this finding, it was found out that students studying in $4^{\text {th }}$ Grade $(\bar{X}=2,84 \pm, 870)$ claimed to get away from the problems of daily life as the reason to watch games in the sub-scale of Escape while watching games more than the students studying in the $1^{\text {st }}$ Grade $(\bar{X}=2,40 \pm, 844)$.

Data analysis also demonstrated that the means of sub-scales on average were found as in the following: seeking excitement and fun ( $\bar{X}=2,98 \pm, 884)$, pride ( $\bar{X}=3,26 \pm 1,002)$, aggression ( $\bar{X}=3,58 \pm 1,022)$, closeness sympathy ( $\bar{X}=2,80 \pm, 899)$, aesthetics ( $\bar{X}=3,14 \pm, 968)$, understanding and knowing $(\bar{X}=2,98 \pm, 985)$, and escape ( $\bar{X}=2,68 \pm, 900$ ) According to that finding, it is obvious that students show more pride and aggressive attitude feelings according to general average of all students.

\section{Discussion and Results}

On this survey, motivations of students in the Faculty of Sports Science at Ataturk University have been investigated and the following result was obtained from the data analysis: We searched the motivation of our students as supporters on this survey, it was found out that motivation levels have increased in the sub dimension of aggression and pride.

This finding may result from that the scores that their team had on the pitch makes the sports fans feel as a matter of honor and thus they adopt more aggressive attitude.

It was also revealed that the urge to participate as an observer to have an experience, to watch the skills of sportsman [9-10], aggression [11], to prove themselves [12], to look for aesthetics [9], and to have fun and competing feeling [13] and sentimental feeling [14] affected their motivations. Considering the variation in the findings of previous studies in the literature, we can say that fans as supporters are motivated by a variety of different sources. The fundamental result of our study was that aggression was the main source of motivation for sports fan in our study especially the supporters who received education on sports science. It is a matter of doubt and dilemma that needs to be taken into consideration as the findings revealed that jobs in which people such as coaches, sports managers, recreation specialists, and sports teachers serve are motivated by 
aggression factor while watching the game.

Considering the gender variable, it was revealed that there was not a statistically significant difference between female and male sports fans in terms of their motivations. More specifically, male sports fans were found to be motivated much more by motives including escape, seeking excitement-fun, pride, sincerity/closeness and aesthetics whereas female sports fans were more motivated by understanding-knowing dimension. Accordingly, female sports fans may have desire to watch matches to have an understanding of match as they generally have much less knowledge compared to male sports fans. In relation to this, this finding seems to support the findings of Wann and Branscombe [15] study which focused on fans and identity in that their study yielded no significant difference between males and females.

As for the analysis of motivation of sports fans in terms of their departments, the findings yielded statistically significant differences among participants. Accordingly, students majoring in Physical Education ( $\overline{\mathrm{X}}=3,38 \pm, 989)$ were more motivated in aesthetic dimension compared to those majoring in Sports Management $(\bar{X}=2,91 \pm, 909)$. This finding may result from that these Physical education students are keener on the game whereas the students majoring in Sports Management are concerned with rivalry.

In terms of participants' grade, there was statistically significant difference among different grades in dimensions of aggression, understanding-knowing and escape. Regarding aggression dimension, the $1^{\text {st }}$ grade students $(\bar{X}=3,88 \pm 1,021)$ had more mean scores compared to the $4^{\text {th }}$ grade students $(\overline{\mathrm{X}}=3,28 \pm 1,064)$. As the $1^{\text {st }}$ grade students are still novices and young, they may not consider the notion of sports as an area of profession. In parallel with this, it was found out that the $4^{\text {th }}$ grade students were found to be the sports fans who were the least motivated by aggression. This finding suggests that the higher the grade is, the lower the level they were motivated by aggression. As for the sub-dimension of escape, the $4^{\text {th }}$ grade students ( $\overline{\mathrm{X}}=2,84 \pm, 870$ ) had more mean scores compared to the $1^{\text {st }}$ grade students $(\bar{X}=2,40 \pm, 844)$. This finding may result from that the $4^{\text {th }}$ grade students prefer to watch games to escape from the stress they feel due to the haste of graduation, exams and concern for the future.

As for understanding-knowing sub-dimension, $4^{\text {th }}$ grade students $(\bar{X}=3,20 \pm, 993)$ had more mean scores than $1^{\text {st }}$ grade students $(\bar{X}=2,70 \pm 1,035)$. Considering this finding, it is possible that $4^{\text {th }}$ and final grade students may prefer to watch games in order to add to their knowledge of their own fields and follow the recent approaches about their area of profession.

In the current research, we searched the motivational aspects of sports science students when they are watching a football match and it was found out that students who have just started at the faculty were motivated by aggression and aggression was both revealed to be the main motive for watching games and found to decrease as grade/ proficiency increases. Moreover, considering the finding that the students who received education on sports at sports science faculty were mainly motivated by aggression is already thought-provoking and needs rethinking and further attention as it is more likely that people who have not even received education on sports at sports science might be more motivated by aggression factor. It is necessary to prevent problems experienced at tribunes to help sports fans feel far from aggression. For that reason, there is a necessity for providing students with the courses that create awareness on these issues to decrease the aggression factor and to prevent students from having such aggression problems. Moreover, we should spread the fair-play spirit among funs and should move them away from aggression.

\section{REFERENCES}

[1] Karaoğlu G.N., Tiryaki, Ş. (2003). A study on developing a scale on motivation of sports fans. Unpublished M.A Thesis. Mersin University, Institute of Health Sciences, Mersin.

[2] Katırc1 H. (2006). Football Club-Fan Communication in Turkey. Anadolu University, Department of Physical Training and Sports, Eskişehir.

[3] Keskin B. (2014). An analysis of behaviors of Football Fans in buying. Unpublished MA Thesis. Süleyman Demirel University, Institute of Social Sciences, Business Department, Isparta.

[4] Baş M. (2008). Fan and Team Identity in Football (Sample of Trabzonspor). Gazi University, Institute of Health Sciences, Department of Physical Training and Sports, Ankara.

[5] Dikici S. T. (2008). Social and Political Profile of Football Fans in Turkey: A sample from "Beşiktaş JK Çarşı" Group. İstanbul.

[6] Kirdar Y. (2006). The effect of marketing communication activities in Institutionalized Sports Clubs on Corporate Image during sports marketing: A sample of Fenerbahçe Sports Club. Ege University, Institute of Social Sciences, Public Relations and Introduction Department. İstanbul.

[7] Cengiz D. (2014). The effect of football and football fans to social events. (Beşiktaş Fan Group: ÇARŞI). Unpublished MA Thesis. Bahçeşehir University, Institute of Social Sciences, Sports Management Department. İstanbul.

[8] Toksöz, C.Why do we love Football? Retrieved from (http://www.cerentokdemir.com/neden-futbolu-seviyoruz.ph p)

[9] Trail, G., \& James, J. (2001). The motivation scale for sport consumption: Assessment of the scale's psychometric properties. Journal of Sport Behaviour, 24(1), 108-127.

[10] Trail, G. T., Robinson, M., Dick, R. ve Gillintine, A. (2003). Motives and points of attachment: Fans versus spectators in intercollegiate athletics. Sport Marketing Quarterly, 12(4), 217-227.

[11] McDonald, M. A., Milne, G. R., \& Hong, J. (2002). Motivational Factors for Evaluating Sport Spectator and 
Participant Markets. Sport Marketing Quarterly, 11(2), 100-13.

[12] Mahony, D. F., Nakazawa, M., Funk, D. C., James, J. D., \& Gladden, J. M. (2002). Motivational factors influencing the behaviour of J. League spectators. Sport Management Review, 5(1), 1-24.

[13] Wann, D. L. (1995). Preliminary validation of the sport fan motivation scale. Journal of Sport \& Social Issues, 19(4), 377-396.
[14] Funk, Daniel C., Lynn L. Ridinger, and Anita M. Moorman. (2003). "Understanding consumer support: Extending the Sport Interest Inventory (SII) to examine individual differences among women's professional sport consumers." Sport Management Review 6.1.p.p.1-31.

[15] Wann, D.L., Branscombe, N.R. (1995).Influence of identification with a sports team on objective knowledge and subjective beliefs, İnternational Journal of Sport Psychology, $26,551-567$ 